\title{
Brücken nach Europa sprengen - nichts für unser Gesundheitswesen
}

\author{
Jürg Schlup
}

Dr. med., Präsident der FMH

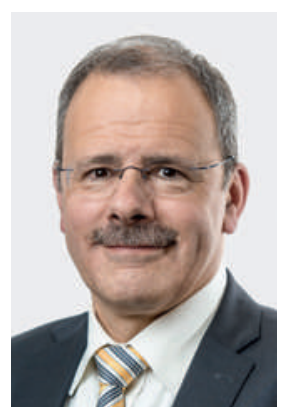

Die am 9. Februar 2014 von der Schweizer Stimmbevölkerung angenommene Initiative "Gegen Masseneinwanderung» stellt mit der darin geforderten Begrenzung der Zuwanderung durch Höchstzahlen und Kontingente unser Gesundheitswesen vor grosse Herausforderungen.

Von den 185000 Beschäftigten unserer Spitäler, Kliniken und Pflegeinstitutionen sind nach Angaben des Spitalverbandes $\mathrm{H}+32 \%$ ausländischer Herkunft [1]. Und da auch unter den ausländischen Mitarbeitern aus demographischen oder anderen Gründen ein ständiger Wechsel stattfindet, benötigen diese Einrichtungen zur Schliessung fluktuationsbedingter Lücken jedes Jahr von neuem etwa 10000 zusätzliche ausländische Gesundheitsfachkräfte [1].

Betrachtet man im Speziellen die Ärzteschaft, zeigt sich auch dort, dass mit 31\% fast ein Drittel aller berufstätigen Ärztinnen und Ärzte ihr Medizinstudium im Ausland absolviert haben. Und auch von den 2014 insgesamt 1501 verliehenen Facharzttiteln gingen 44\% an Ärztinnen mit einem ausländischen Diplom [2]. All diesen Kolleginnen und Kollegen möchte ich für ihren hochqualifizierten und unverzichtbaren Beitrag zu unserer Gesundheitsversorgung herzlich danken!

\section{Die Umsetzung der Masseneinwanderungs- initiative ist eine grosse Herausforderung für das Schweizer Gesundheitswesen.}

Die Abhängigkeit unserer Gesundheitsversorgung von ausländischen Fachkräften ist bedeutend und der Mangel an Ärzten und anderen Gesundheitsfachpersonen, der bereits heute in einigen Regionen und Fachrichtungen besteht, dürfte uns in einigen Jahren als Luxusproblem erscheinen, sollte die Umsetzung der Masseneinwanderungsinitiative $\mathrm{zu}$ eng ausgelegt werden. Denn die Hoffnung, den Auswirkungen der Initiative durch eine bessere Ausschöpfung des inländischen Fachkräftepotentials begegnen zu können, dürfte sich im Gesundheitsbereich bestenfalls langfristig erfüllen. Selbst wenn man deutlich optimistischere Annahmen träfe als bestehende Schätzungen [1], bliebe das Ergebnis dasselbe: Die im Inland potentiell verfügbaren
Fachpersonen können den Personalbedarf kurz- und mittelfristig bei weitem nicht decken.

Auch wenn der Braindrain in die Schweiz allein aus ethischen Gründen so nicht fortbestehen darf, erfordern viele der in der Gesundheitsversorgung nachgefragten spezifischen Qualifikationen doch längere Vorlaufzeiten, um ausreichend inländische Fachkräfte ausbilden zu können. Möchten wir nicht hinter unsere bewährten Qualitätsstandards zurückfallen, wird der

31\% der in der Schweiz tätigen Gesundheitsfachkräfte stammen aus dem Ausland. Sie leisten einen unverzichtbaren Beitrag für die Versorgung der Bevölkerung.

Weg von der Matura bis zur eigenverantwortlich tätigen Fachärztin weiterhin etwa elf Jahre dauern. Viel stärker als die lange Aus- und Weiterbildungszeit fällt jedoch die Trägheit der Politik ins Gewicht. So werden wir trotz Erhöhung der Studienplatzzahlen seit 2008 erst ab 2020 in der Humanmedizin wieder die Diplomzahlen von 1980 erreichen. Und die vom Bundesrat 2011 empfohlenen 1200 bis 1300 Studienabschlüsse pro Jahr werden wir frühestens ab 2025 erreichen. Die Initiative muss allerdings bereits in 18 Monaten umgesetzt werden.

Damit die Versorgungssicherheit trotz der Masseneinwanderungsinitiative gewährleistet bleibt, setzt sich die FMH gemeinsam mit Spitälern und anderen Berufsverbänden des Gesundheitswesens für eine praxistaugliche Umsetzung ein. So könnte bspw. eine Schutz- oder Ventilklausel die zusätzliche administrative Belastung des Gesundheitswesens reduzieren. Auch langfristigen Anstellungen darf nichts im Wege stehen, vor allem um Patienten Qualität und dem dringend benötigten Personal attraktive Bedingungen bieten zu können. Vor allem aber sollten die Kantone ausreichend Spielraum zur Umsetzung erhalten, um ihrem Versorgungsauftrag gerecht werden und Einschränkungen der Gesundheitsversorgung abwenden zu können. - Zu Risiken und Nebenwirkungen fragen Sie Ihren politischen Volksvertreter, aber bitte vor dem 18. Oktober. 